\title{
Experimental investigation of secondary fast neutrons produced in carbon ion radiotherapy
}

\author{
D. Schardt ${ }^{\star}$, H. Iwase, R.S. Simon \\ Gesellschaft für Schwerionenforschung \\ Planckstr.1, D-64291 Darmstadt, Germany \\ E-mail: d.schardt@gsi.de
}

\section{K. Gunzert-Marx}

Siemens Medical Solutions, Particle Therapy

D-91052 Erlangen, Germany

E-mail: konstanze.gunzert-marx@siemens.com

The radiation field of heavy-ion beams used for radiotherapy applications is significantly affected by nuclear fragmentation processes along the penetration path in tissue, causing an attenuation of the primary ion beam flux and the build-up of light fragments including fast neutrons. The emission of secondary fragments from $200 \mathrm{MeV} / \mathrm{u}$ carbon ions delivered from the synchrotron SIS-18 at GSI was investigated using a water absorber as tissue-equivalent beam stopping target. Energy spectra, angular distributions and yields of fast neutrons and charged particles emerging from the absorber downstream were investigated by using fast scintillation detectors and time-of-flight techniques. From the resulting yield of 0.54 neutrons $\left(\mathrm{E}_{\mathrm{n}}>10 \mathrm{MeV}\right)$ per primary ${ }^{12} \mathrm{C}$ ion a neutron dose of $5.4 \mathrm{mSv}$ per Cobalt Gray Equivalent (GyE) delivered to the target volume is estimated. Additional measurements employing a wide-energy neutron detector (WENDI-II) under identical irradiation conditions were found to be in fair agreement with the dose estimate based on the measured fast neutron fluences.

International Workshop on Fast Neutron Detectors

University of Cape Town, South Africa

April 3 - 6, 2006

\section{${ }^{*}$ Speaker}




\section{Introduction}

Carbon ions offer favorable conditions for the treatment of deep-seated, localized tumors due to their physical and biological properties. Their dose profile is - in contrast to photons - inverted, the dose maximum is deposited at the end of the particle range in the so called "Bragg peak". In addition the relative biological effectiveness (RBE) of carbon ions increases in the region of the Bragg peak by a factor of three to five. Nuclear reactions along the penetration path, however, cause a significant alteration of the radiation field. At energies of several hundred $\mathrm{MeV} / \mathrm{u}$ which are required for radiotherapy the most frequent nuclear interactions are peripheral collisions where the beam particles loose one or several nucleons. The fragments continue travelling with nearly the same velocity and direction. These nuclear reactions lead to an attenuation of the primary beam flux and a build-up of lower- $Z$ fragments with increasing penetration depth. As the range of the particles scales with $\mathrm{A} / \mathrm{Z}^{2}$ the depth-dose profile of heavy-ion beams shows a characteristic fragment tail beyond the Bragg peak (Fig.1).
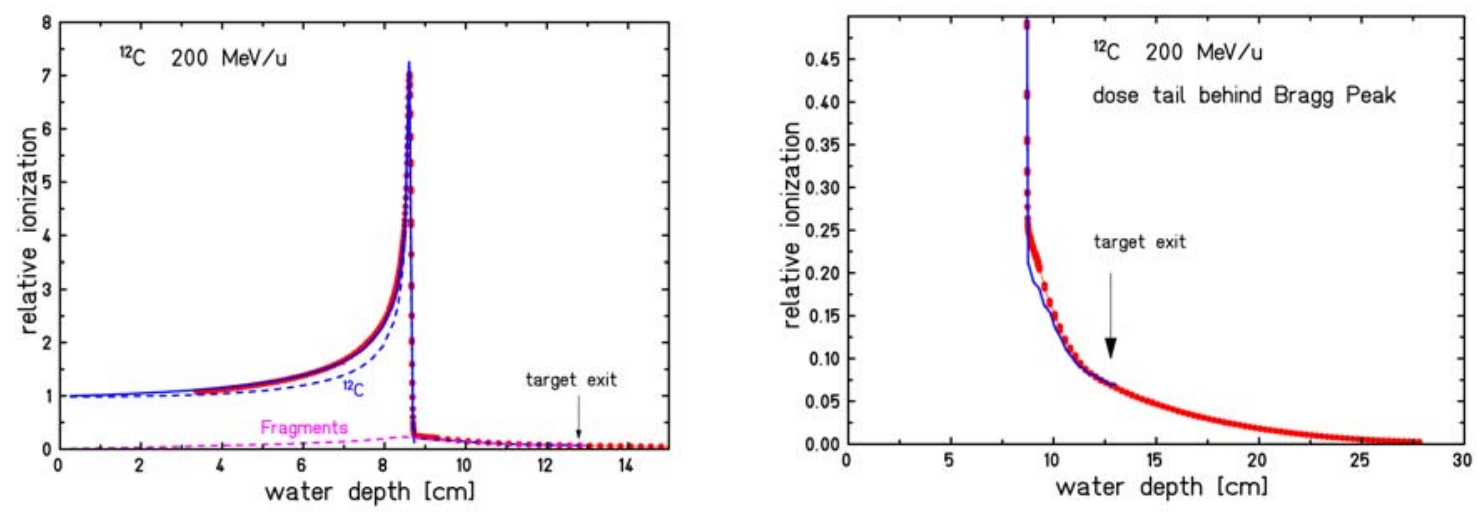

Figure 1: Bragg curve of $200 \mathrm{MeV} / \mathrm{u}^{12} \mathrm{C}$ ions in water. The data points (red) are shown together with a Monte-Carlo calculation using the PHITS code [1]. The calculated contributions of primary ions and secondary fragments are indicated. The right part shows a magnified view of the dose tail behind the Bragg peak.

The attenuation of the primary particle flux and build-up of lower- $\mathrm{Z}$ charged fragments along the penetration path in water were studied earlier at LBL Berkeley [2], at GSI Darmstadt [3] and at the HIMAC facility at Chiba [4]. For the production of neutrons in thick targets, however, the experimental data are rather scarce and mainly concern the design of shielding components for accelerators or space missions. Neutron production by fragmentation of light ions, like helium ions in water was investigated by Cecil et al.[5] and more recently, neutrons produced from carbon ion beams stopped in graphite targets by Kurosawa et al.[6]. Here we report on measurements [7] of the fast neutron component produced by nuclear fragmentation of carbon ions in a thick water target and their dose contribution in patient treatment irradiations. 


\section{Spectral measurements of fast neutrons produced from $200 \mathrm{MeV} / \mathrm{u}^{12} \mathrm{C}$ ions}

A pencil-like beam of $200 \mathrm{AMeV}{ }^{12} \mathrm{C}$ ions was stopped in a $12.78 \mathrm{~cm}$ thick water target. The range of the primary particles is $8.6 \mathrm{~cm}$, corresponding to $67 \%$ of the target thickness (see Fig.1). Light beam fragments emerging in forward direction from the water target were detected by a $\Delta$ E-E-telescope in about $3 \mathrm{~m}$ distance from the target and at angles between $0^{\circ}$ to $30^{\circ}$ degrees with respect to the beam axis. The telescope consisted of a $14 \mathrm{~cm}$ long, hexagonally shaped $\mathrm{BaF}_{2}$ scintillator and a $9 \mathrm{~mm}$ thick NE102 scintillator (veto detector) in front to discriminate charged particle and neutron events. The veto scintillator had an inscribed radius of $5.4 \mathrm{~cm}$, the $\mathrm{BaF}_{2}$ crystal of $4.5 \mathrm{~cm}$. Particle energies were measured by time-of-flight techniques. A thin NE102 scintillator in front of the target counted the number of the incoming ${ }^{12} \mathrm{C}$ ions and delivered the start signal for the time-of flight measurement. The stop signal was obtained from the $\mathrm{BaF}_{2}$ detector. The nuclear charge and mass of the particles were identified by the correlation of their energy lost in the veto scintillator and their energy deposited in the $\mathrm{BaF}_{2}$ detector. The neutron efficiency of the $\mathrm{BaF}_{2}$ detector was obtained from separate measurements [8] using quasi-monoenergetic neutron beams at UCL Louvain (Belgium) and iThemba LABS (South Africa).

The energy spectra and angular distributions of neutrons emerging from the water target at angles of $0^{\circ}, 5^{\circ}, 10^{\circ}, 20^{\circ}$ and $30^{\circ}$ are shown in Figs. 2 and 3. Neutrons are mainly emitted in forward direction and a broad maximum can be observed at about half of the initial beam energy at small angles $\left(0^{\circ}\right.$ and $\left.5^{\circ}\right)$, indicating a strong contribution of projectile abrasion in peripheral collisions. The spectra measured at $10^{\circ}, 20^{\circ}$ and $30^{\circ}$ have an exponential shape, which is typical for individual high energy nucleons and fragments evaporated from the hot reaction zone. From $0^{\circ}$ to $30^{\circ}$ a significant fraction of the neutron energy spectra extends to energies greater than 200 $\mathrm{MeV} / \mathrm{u}$, the energy of the incident carbon ions.

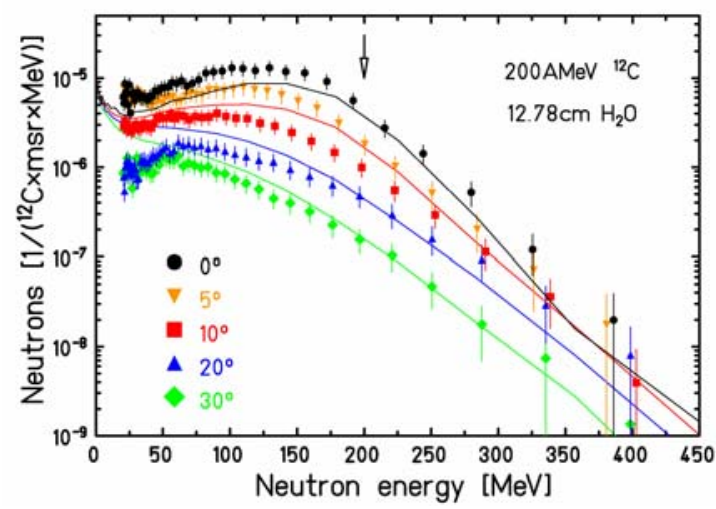

Figure 2: Neutron energy spectra measured at various angles with respect to the beam axis. The arrow indicates the energy of the primary ${ }^{12} \mathrm{C}$ ions. The solid lines represent calculations with the Monte-Carlo code PHITS [1].

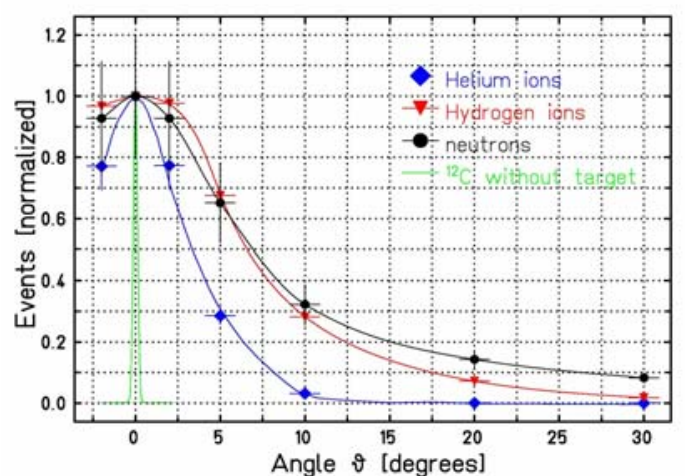

Figure 3: Angular distributions of fast neutrons and charged fragments (normalized at $0^{\circ}$ ) obtained by integration of the measured energy spectra. 
In order to obtain the yields of secondary neutrons and charged fragments in the forward hemisphere the angular distributions were extrapolated to $90^{\circ}$ and integrated. The results are shown in Table 1 together with estimated doses values (see section 3).

\begin{tabular}{lcc}
\hline & Yield $\left(\right.$ per $^{12} \mathrm{C}$ ion $)$ & Dose $\left[\mathrm{mGy} \mathrm{Gy}^{-1}\right]$ \\
\hline Neutrons & $\mathbf{0 . 5 4} \pm \mathbf{0 . 2 0}$ & $\mathbf{8} \pm \mathbf{3}$ \\
Protons & $0.20 \pm 0.05$ & $24 \pm 6$ \\
Hydrogen & $0.31 \pm 0.07$ & $37 \pm 8$ \\
Helium & $0.13 \pm 0.03$ & $62 \pm 14$ \\
\hline
\end{tabular}

Table 1: Total particle yields $(\mathrm{E}>10 \mathrm{MeV})$ in the forward hemisphere and the corresponding estimated dose per treatment Gray.

\section{Estimation of the neutron dose in patient treatments with carbon ions}

Based on the measured neutron yield for $200 \mathrm{MeV} / \mathrm{u}{ }^{12} \mathrm{C}$ ions stopping in water the dose contribution of secondary fast neutrons in patient treatments with carbon ions was estimated by converting the neutron fluence into kerma (kinetic energy released per mass unit). As a typical treatment volume the irradiation of a cube with the side length of $5 \mathrm{~cm}$, i.e. an irradiated area of $25 \mathrm{~cm}^{2}$, was assumed. To apply $20 \mathrm{~Gy}$ absorbed dose (corresponding to about $60 \mathrm{GyE}$ biologically effective dose) to the given volume, a total amount of $5 \cdot 10^{10}$ carbon ions is required. This number is obtained from the computer code TRiP [9] which is routinely used for patient treatment planning at GSI. The biologically effective dose given in units of Cobalt Gray Equivalent (GyE) includes the relative biological effectiveness (RBE) of the particles. Because of the complex composition of the particle field due to nuclear fragmentation, however, the biologically effective dose is not a simple product of absorbed dose and a mean RBE value but is evaluated by an iterative procedure using the TRiP code [10].

Taking the neutron yield of $0.54 /$ ion, the neutron fluence $4 \mathrm{~cm}$ behind the irradiated volume then amounts to $(1.1 \pm 0.4) \cdot 10^{9}$ neutrons $/ \mathrm{cm}^{2}$. (The distance of $4 \mathrm{~cm}$ corresponds to the difference of target thickness and ion beam range in our measurements.) The kerma coefficient in water recommended in the ICRP 63 was extrapolated to a neutron energy of $200 \mathrm{MeV}$ $\left(\mathrm{k}=150 \mathrm{pGy} \cdot \mathrm{cm}^{2}\right)$ and multiplied with the fluence $\Phi(E)$ to obtain the neutron kerma. The resulting neutron dose of $8 \mathrm{mGy}$ per treatment-Gy (table 1) is less then $1 \%$ of the treatment dose. This result is in good agreement with recent calculations [11] based on the Monte-Carlo code GEANT4.

Alternatively, the neutron dose equivalent (as defined in medical physics) can be estimated by multiplying the neutron fluence with the conversion coefficient $h_{\Phi}(E)$. Using a mean value of $\mathrm{h}_{\Phi}=300 \mathrm{pSv} \cdot \mathrm{cm}^{2}$ [12] we obtain a dose value of $16 \mathrm{mSv}$ per treatment-Gy or $5.4 \mathrm{mSv}$ per treatment-GyE.

Using the Wide-Energy Neutron Detection Instrument (WENDI-II) [13] the neutron dose and angular distribution was obtained in independent measurements under the same experimental conditions (beam, target, geometry) as for the fluence measurements described 
above. At small angles below $10^{\circ}$ the dose values obtained from the WENDI measurements are higher by about a factor of 2 compared to the dose values estimated from the fluence measurements. This might be explained by the response of WENDI to high-energy protons and alpha particles which are also present in the particle field and can not be easily corrected for.

\section{Comparison of neutron doses in proton and carbon ion therapy}

In table 2 dose contributions of secondary neutrons obtained from the present work with ${ }^{12} \mathrm{C}$ ions at GSI and from measurements at the proton therapy facility at PSI (Switzerland) [14] are compared. Both treatment units use scanning beam delivery systems. Although the yield of fast neutrons is much higher for ${ }^{12} \mathrm{C}$ ions than for protons the neutron doses per treatment-GyE are not very different because of the much lower number of ${ }^{12} \mathrm{C}$ ions needed to produce the same biologically effective dose as protons.

\begin{tabular}{lcccc}
\hline & $\begin{array}{c}\text { Beam } \\
{[\mathrm{MeV} / \mathrm{u}]}\end{array}$ & $\begin{array}{c}\text { Target } \\
{[\mathrm{g} / \mathrm{cm} 2]}\end{array}$ & $\begin{array}{c}\text { Neutrons per } \\
\text { primary ion }\end{array}$ & $\begin{array}{c}\text { Neutron dose* } \\
{[\mathrm{mSv} \text { per treatment-GyE] }}\end{array}$ \\
\hline GSI [7] & ${ }^{12} \mathrm{C} \mathrm{(200)}$ & $\mathrm{H}_{2} \mathrm{O}(12.78)$ & $0.54 \pm 20 \%$ & $5.4 \pm 20 \%$ (scanning) \\
Schneider [14] & $\mathrm{p}(177)$ & $\mathrm{H}_{2} \mathrm{O}(30.1)$ & $(0.025)$ & $2.3 \pm 30 \%$ (scanning) \\
\hline
\end{tabular}

* Neutron dose expected in healthy tissue of the patient in the non-treated volume for a medium-sized target

Table 2: Comparison of neutron yields and neutron doses in ${ }^{12} \mathrm{C}$ ion and proton radiotherapy for scanning beam delivery systems. The yield number given in parentheses was recalculated from the dose.

For passive beam delivery systems, where secondary neutrons are produced mainly by interactions of the primary beam with collimators, range shifters and other elements located in front of the patients, the neutron dose varies significantly with the configuration of the beam modifying equipment. This was examined experimentally by Yan et al. [15] for the medical beam line at the Harvard Cyclotron Laboratory. They found neutron dose equivalent values of typically $1-5 \mathrm{mSv} / \mathrm{Gy}$ at $50 \mathrm{~cm}$ distance from isocenter.

Monte Carlo calculations performed by Agosteo et al. [16] for the proton therapy beam lines at PSI (active scanning) and at iThemba LABS South Africa (passive system) confirm the experimental results by Schneider et al. [14] and Binns et al. [17]. The calculations indicate a factor of 10 higher secondary dose for the passive beam delivery system which seems to originate predominantly from neutrons generated in the last collimator.

In conclusion, the present work has shown that the dose contribution caused by secondary neutrons in carbon ion radiotherapy is of the order of $1 \%$ of the treatment dose and much lower than the dose of secondary charged fragments. Further measurements as well as Monte Carlo 
calculations are planned to investigate the dose deposition of secondary fragments, in particular in regions outside of the planned treatment volume.

\section{References}

[1] H. Iwase et al., Calculation of secondary neutron fields generated by high-energy heavy-ion reactions using the Monte-Carlo code PHITS, contribution to this conference.

[2] W. Schimmerling et al., Fragmentation of $670 \mathrm{~A} \mathrm{MeV} \mathrm{Neon} 20$ as a function of depth in water, Radiat. Res. 120 (1989) 36.

[3] I. Schall I. et al., Charge-changing nuclear reactions of relativistic light-ion beams ( $5 \leq Z \leq 10$ ) passing through thick absorbers, Nucl. Instr. and Meth. B117 (1996) 221.

[4] N. Matsufuji et al., Spatial fragment distribution from a therapeutic pencil-like carbon beam in water, Phys. Med. Biol. 50 (2005) 3393.

[5] R.A. Cecil et al., Neutron angular and energy distributions from 710-MeV alphas stopping in water, carbon and lead and $640 \mathrm{MeV}$-alphas stopping in lead, Phys. Rev. C21 (1980) 2471.

[6] T. Kurosawa et al., Measurements of secondary neutrons produced from thick targets bombarded by high-energy helium and carbon ions, Nucl., Sci. Eng. 132 (1999) 30.

[7] K. Gunzert-Marx et al., Fast neutrons produced by nuclear fragmentation in treatment irradiations with ${ }^{12} \mathrm{C}$ beam, Rad. Prot. Dosim. 110 (2004) 595.

[8] K. Gunzert-Marx et al., Response of a $\mathrm{BaF}_{2}$ scintillation detector to quasi-monoenergetic fast neutrons in the range of 45 to $198 \mathrm{MeV}$, Nucl. Instrum. Meth. A536 (2005) 146.

[9] M. Krämer, O. Jäkel, T. Haberer, G. Kraft, D. Schardt, U. Weber, Treatment planning for heavyion radiotherapy: physical beam model and dose optimization, Phys. Med. Biol. 45 (2000) 3299.

[10] M. Krämer and M. Scholz, Treatment planning for heavy-ion radiotherapy: calculation and optimization of biologically effective dose, Phys. Med. Biol. 45 (2000) 3319.

[11] I. Pshenichnov et al., Neutrons from fragmentation of light nuclei in tissue-like media: a study with the GEANT4 toolkit, Phys. Med. Biol. 50 (2005) 5493.

[12] A. Ferrari, M. Pellicioni, Fluence to dose equivalent conversion data and effective quality factors for high energy neutrons, Rad. Prot. Dosim. 76 (1998) 215.

[13] R.H. Olscher et al., WENDI: An improved neutron rem meter, Health Physics 79 (2000) 170.

[14] U. Schneider et al., Secondary neutron dose during proton therapy using spot scanning, Int. J. Radiation Oncology Biol. Phys. 53 (2002) 244.

[15] X. Yan, U. Titt, A.M. Koehler, W.D. Newhauser, Measurement of neutron dose equivalent to proton therapy patients outside of the proton radiation field, Nucl. Instrum. Meth. A476 (2002) 429.

[16] S. Agosteo et al., Secondary neutron and photon dose in proton therapy, Radiother. Oncol. 48 (1998) 293.

[17] P.J. Binns, J.H. Hough, Secondary dose exposures during $200 \mathrm{MeV}$ proton therapy, Rad. Prot. Dosim. 70 (1997) 441. 